\title{
COMPACTIFICATION OF STRONGLY COUNTABLE DIMENSIONAL SPACES ${ }^{1}$
}

\author{
BY ARLO W. SCHURLE
}

Communicated by R. H. Bing, May 22, 1967

In this paper all spaces, including compactifications, are separable metrizable. Recall the following definitions. A space $X$ is strongly countable dimensional if $X$ is a countable union of closed finitedimensional subsets. $X$ is a $G_{\delta}$ space if $X$ is a $G_{\delta}$-set in each space in which it is topologically embedded. A space $Y$ is a pseudo-polytope if $Y=\Sigma_{1} \cup \Sigma_{2} \cup \ldots$, where each $\Sigma_{i}$ is a simplex, $\Sigma_{i} \cap \Sigma_{j}$ is either empty or a face of both $\Sigma_{i}$ and $\Sigma_{j}$, and diam $\Sigma_{i} \rightarrow 0$ as $i \rightarrow \infty$. The term map always denotes a continuous function. Other notation is as in [3] and $[8]$.

In [5] Lelek proved that every $G_{\delta}$-space $X$ has a compactification $d X$ such that $d X \backslash X$ is a pseudo-polytope. He then raised the question of whether every strongly countable dimensional $G_{\delta}$ space $X$ has a strongly countable dimensional compactification. This paper answers that question in the affirmative. We first state some preliminary propositions.

Proposition 1. Let $M \subset X$ with $\operatorname{dim} M \leqq n$, and let $\left\{U_{i} \mid i=1,2, \cdots\right\}$ be a sequence of sets open in $X$ and covering $M$. Then there is a sequence $\left\{V_{i} \mid i=1,2, \cdots\right\}$ of sets open in $X$ and covering $M$ such that ord $\left\{V_{i} \mid i=1,2, \cdots\right\} \leqq n+1$ and such that $V_{k(n+1)+j} \subset U_{k+1}$ for $k=0,1,2, \cdots$ and $j=1,2, \cdots, n+1$.

Proof. The proof involves only a slight extension of the argument on page 54 of [2].

Proposition 2. Let $G$ be an open subset of a totally bounded space $Y$, and let $M_{1}, M_{2}, \cdots, M_{r}$ be relatively closed subsets of $G$ with $\operatorname{dim} M_{i}=m_{i}<\infty$ for $i=1,2, \cdots, r$. Let $\epsilon>0$. Then there is a collection $\left\{G_{i} \mid i=1,2, \cdots\right\}$ such that $G=\cup_{i=1}^{\infty} G_{i}$ and

(i) Each $G_{i}$ is open in $Y$.

(ii) $\left\{G_{i} \mid i=1,2, \ldots\right\}$ is star-finite.

(iii) $\bar{G}_{i} \subset G$ for $i=1,2, \ldots$.

1 This paper was written in partial fulfillment of the requirements for the degree of Doctor of Philosophy. 
(iv) $\operatorname{diam} G_{i}<\epsilon$ for $i=1,2, \ldots$ and $\operatorname{diam} G_{i} \rightarrow 0$ as $i \rightarrow \infty$.

(v) $\operatorname{ord}\left\{G_{i} \mid G_{i}\right.$ meets $\left.M_{1} \cup M_{2} \cup \ldots \cup M_{k}\right\} \leqq m_{1}+1+m_{2}+1+\cdots$ $+m_{k}+1$ for $k=1,2, \cdots, r$.

Proof. We sketch the proof of this proposition. From page 114 of [4] we get a collection $\left\{G_{i}^{\prime} \mid i=1,2, \cdots\right\}$ satisfying (i)-(iv). Open covers satisfying (i)-(iv) and (v) for $k=1,2, \cdots, r$ are now defined inductively. By Proposition 1 there is a sequence $\left\{V_{i} \mid i=1,2, \ldots\right\}$ of open sets covering $M_{1}$ such that ord $\left\{V_{i} \mid i=1,2, \ldots\right\} \leqq m_{1}+1$ and $V_{k(n+1)+j} \subset G_{k+1}^{\prime}$ for $k=0,1,2, \cdots$ and $j=1,2, \cdots, m_{1}+1$. The collection $\left\{V_{i} \mid i=1,2, \cdots\right\} \cup\left\{G_{i}^{\prime} \backslash M_{1} \mid i=1,2, \cdots\right\}$ then satisfies (i)-(iv) and (v) for $k=1$.

Suppose $\left\{G_{i}^{\prime} \mid i=1,2, \cdots\right\}$ covers $G$, satisfies (i)-(iv) and (v) for each $k=1,2, \cdots, n$. Let $C=M_{1} \cup M_{2} \cup \cdots \cup M_{n}$. By Proposition 1 there is a sequence $\left\{V_{i} \mid i=1,2, \cdots\right\}$ of open sets covering $M_{n+1} \backslash C$ such that ord $\left\{V_{i} \mid i=1,2, \cdots\right\} \leqq m_{n+1}+1$ and $V_{k(n+1)+j} \subset G_{k+1}^{\prime} \backslash C$ for $k=0,1,2, \ldots$ and $j=1,2, \cdots, m_{n+1}+1$. The collection $\left\{G_{i}^{\prime} \backslash\left(C \cup M_{n+1}\right) \mid i=1, \quad 2, \cdots\right\} \cup\left\{V_{i} \mid i=1, \quad 2, \cdots\right\} \cup\left\{G_{i}^{\prime} \mid G_{i}^{\prime}\right.$ meets $C\}$ satisfies (i)-(iv) and (v) for each $k=1,2, \cdots, n+1$. This completes the inductive step and the sketch of the proof.

We are now in a position to prove the first theorem.

TheORem 1. Let $C$ be a closed subset of a compact space $Y$, and let $M_{1}, M_{2}, \cdots, M_{r}$ be closed subsets of $Y$ with $\operatorname{dim} M_{i}=m_{i}<\infty$ for $i=1,2, \cdots, r$. Let $\epsilon>0$. Then there is an $\epsilon-m a p f: Y \rightarrow I^{\omega}$ such that $f(C) \cap f(Y \backslash C)=\varnothing, f \mid C$ is a homeomorphism, $f(Y \backslash C)$ is a countable polytope $P$, and $\operatorname{dim} f\left(M_{i} \backslash C\right) \leqq m_{1}+1+m_{2}+1+\cdots+m_{i}$ for $i=1,2$, $\cdots, r$. Further, $P=\Sigma_{1} \cup \Sigma_{2} \cup \ldots$ where each $\Sigma_{i}$ is a simplex and $\operatorname{diam} \Sigma_{i} \rightarrow 0$ as $i \rightarrow \infty$.

Proof. We may assume that $Y \subset I^{\omega}$ and that the first coordinate of each point of $Y$ is zero. Let $\mathcal{G}=\left\{G_{i} \mid i=1,2, \cdots\right\}$ be the open cover of $Y \backslash C$ given by Proposition 2 with $\operatorname{diam} G_{i}<\epsilon / 8$ for $i=1,2, \ldots$. For each $i$ such that $G_{i} \neq \varnothing$ pick a point $g_{i} \in G_{i}$. Then pick points $p_{i}$ with first coordinate greater than zero such that $d\left(p_{i}, g_{i}\right)$ $<\min \{1 / i, \epsilon / 8\}$ and such that $\left\{p_{i} \mid i=1,2, \ldots\right\}$ is in general position. Let $N$ be the collection of simplexes spanned by finite subsets $\left\{p_{i_{0}}, p_{i_{1}}, \cdots, p_{i_{n}}\right\}$ where $G_{i_{0}} \cap G_{i_{1}} \cap \cdots \cap G_{i_{n}} \neq \varnothing$. The points $\left\{p_{i} \mid i=1,2, \cdots\right\}$ may be picked in such a way that $N$ is a CWpolytope, and certainly $N \cap Y=\varnothing$. Also, $N=\Sigma_{1} \cup \Sigma_{2} \cup \ldots$ where each $\Sigma_{i}$ is a simplex and diam $\Sigma_{i} \rightarrow 0$ as $i \rightarrow \infty$. Define $f^{\prime}: Y \rightarrow I^{\omega}$ by 


$$
\begin{gathered}
\text { if } x \in C, \\
f^{\prime}(x)=\left\{\begin{array}{l}
\sum_{i=1}^{\infty} d\left(x, Y \backslash G_{i}\right) p_{i} \\
\sum_{i=1}^{\infty} d\left(x, Y \backslash G_{i}\right)
\end{array} \text { if } x \in C .\right.
\end{gathered}
$$

It is not hard to show that $f^{\prime}$ is continuous, and that $d\left(z, f^{\prime}(z)\right)$ $<\epsilon / 4$ for each $z \in Y$. Triangulate $N$ into simplexes of diameter less than $\epsilon / 4$. By a suitable induction, a map $f_{1}: f^{\prime}(Y) \cap N \rightarrow N$ may be defined in such a way that $f^{\prime}(y)$ and $f_{1} f^{\prime}(y)$ are in the same simplexes and $f_{1}\left(f^{\prime}(Y) \cap N\right)$ is a subpolytope $P$ of $N$. The map $f: Y \rightarrow I^{\omega}$ defined by

$$
f(z)= \begin{cases}z & z \in C, \\ f_{1} f^{\prime}(z) & z \in Y \backslash C\end{cases}
$$

is then an $\epsilon$-map such that $f(C) \cap f(Y \backslash C)=\varnothing, f \mid C$ is a homeomorphism, and $f(Y \backslash C)$ is the desired polytope $P$. Finally, let $y \in M_{i} \backslash C$. By the conditions on the cover $\mathcal{G}, y$ is in at most $m_{1}+1+m_{2}+1+\ldots$. $+m_{i}+1$ elements of $g$. Thus $f^{\prime}(y)$, and hence also $f_{1} f^{\prime}(y)$, is in a simplex of dimension not greater than $m_{1}+1+m_{2}+1+\cdots+m_{i}$. Since $P$ is a countable polytope, $\operatorname{dim} f\left(M_{i} \backslash C\right) \leqq m_{1}+1+m_{2}+1$ $+\cdots+m_{i}$. Q.E.D.

Theorem 1 now enables us to prove our main theorem.

THEOREM 2. Let $X$ be a strongly countable dimensional $G_{\delta}$ space. Then there is a strongly countable dimensional compactification $d X$ of $X$ such that $d X \backslash X$ is a pseudo-polytope.

Proof. Let $X=F_{1} \cup F_{2} \cup \ldots$ where $F_{i}$ is closed and $\operatorname{dim} F_{i}$ $=m_{i}<\infty$ for $i=1,2, \ldots$. By a result of Hurewicz [1] there is a compactification $c X$ of $X$ such that $\operatorname{dim} \bar{F}_{i}^{c X}=m_{i}$ for $i=1,2, \cdots$. Let $n_{i}=m_{1}+1+m_{2}+1+\cdots+m_{i}$. Since $X$ is a $G_{\delta}$ space, $c X \backslash X$ $=Y_{1} \cup Y_{2} \cup \ldots$ where each $Y_{i}$ is compact and $Y_{i} \subset Y_{i+1}$ for $i=1,2, \cdots$. Let $Y_{0}=\varnothing$. By Theorem 1 there is a $1 / i$-map $f_{i}: Y_{i} \rightarrow I^{\omega}$ such that $f_{i}\left(Y_{i-1}\right) \cap f_{i}\left(Y_{i} \backslash Y_{i-1}\right)=\varnothing, f_{i} \mid Y_{i-1}$ is a homeomorphism, $f_{i}\left(Y_{i} \backslash Y_{i-1}\right)$ is a countable polytope $P$, and $\operatorname{dim} f_{i}\left(\bar{F}_{k}^{c X} \cap\left(Y_{i} \backslash Y_{i-1}\right)\right)$ $\leqq n_{k}$ for $k=1,2, \cdots, i$.

Decompose $c X$ into sets $f_{i}^{-1}(z)$ for $z \in f_{i}\left(Y_{i} \backslash Y_{i-1}\right)$ and into individual points $x \in X$. Let the quotient space be $d X$ and let $f: c X \rightarrow d X$ be the quotient map. It may be shown that the decomposition of $c X$ is upper semicontinuous, so that $f$ is a closed map. Hence $d X$ is a 
compactification of $X$. Further, it is easily shown that there is a uniformly continuous homeomorphism $g_{i}: f_{i}\left(Y_{i} \backslash Y_{i-1}\right) \rightarrow f\left(Y_{i} \backslash Y_{i-1}\right)$. Since $f_{i}\left(Y_{i} \backslash Y_{i-1}\right)$ is a countable polytope for $i=1,2, \cdots, d X \backslash X$ is a pseudo-polytope.

To show that $d X$ is strongly countable dimensional it is enough to show that $\bar{F}_{i}^{d X}$ is strongly countable dimensional for $i=1,2, \cdots$. Fix a positive integer $k$. Since $f$ is a closed map, $\bar{F}_{k}^{d X}=f\left(\bar{F}_{k}^{c X}\right)=F_{k}$ $\cup \cup_{i=1}^{\infty} f\left(\bar{F}_{k}^{c X} \cap\left(Y_{i} \backslash Y_{i-1}\right)\right)$. Also, $f\left(\bar{F}_{k}^{c X} \cap Y_{k-1}\right) \subset f(c X \backslash X)=d X \backslash X$, so $f\left(\bar{F}_{k}^{c X} \cap Y_{k-1}\right)$ is strongly countable dimensional. Let $C_{n}$ $=\bigcup_{j=k}^{n} f\left(\bar{F}_{k}^{c X} \cap\left(Y_{j} \backslash Y_{j-1}\right)\right)$ for $n=k, k+1, \cdots$ and let $D_{k}=\cup_{j=k}^{\infty} C_{j}$. Each $C_{j}$ is closed in $D_{k}$. Further, $\operatorname{dim} C_{k}=\operatorname{dim} f\left(\bar{F}_{k}^{c X} \cap\left(Y_{k} \backslash Y_{k-1}\right)\right) \leqq n_{k}$. Suppose $\operatorname{dim} C_{i} \leqq n_{k}$. Then $C_{i+1}=C_{i} \cup f\left(\bar{F}_{k}^{c X} \cap\left(Y_{i+1} \backslash Y_{i}\right)\right), C_{i}$ is closed in $C_{i+1}$, and $\operatorname{dim} f\left(\bar{F}_{k}^{c X} \cap\left(Y_{i+1} \backslash Y_{i}\right)\right) \leqq n_{k}$, so $\operatorname{dim} C_{i+1} \leqq n_{k}$. Therefore $\operatorname{dim} D_{k} \leqq n_{k}$, and $\operatorname{dim} D_{k} \cup F_{k} \leqq n_{k}+m_{k}+1 . D_{k} \cup F_{k}$ is open in $\bar{F}_{k}^{d X}$, so by Proposition $2 D_{k} \cup F_{k}=\cup_{i=1}^{\infty} G_{k i}$, where $\bar{G}_{k i}^{d X} \subset D_{k} \cup F_{k}$ for $i=1,2, \cdots$. Hence $\operatorname{dim} \bar{G}_{k i}^{d X} \leqq n_{k}+m_{k}+1$, and $\bar{F}_{k}^{d X}$ is strongly countable dimensional. Q.E.D.

Sklyarenko gives an example in [9] which shows that being a $G_{\delta}$ space is a necessary hypothesis in Theorem 2.

\section{BIBLIOGRAPHY}

1. W. Hurewicz, Über Einbettung separabler Räume in gleichdimensionale kompakte Räume, Monatshefte für Mathematik und Physik 37 (1930), 199-208.

2. W. Hurewicz and H. Wallman, Dimension theory, Princeton Univ. Press, Princeton, N. J., 1941.

3. J. L. Kelley, General Topology, Van Nostrand, Princeton, N. J., 1955.

4. Casimir Kuratowski, Topologie. I, 3rd ed., Monogr. Mat. Warsaw, 1952.

5. A. Lelek, On dimension of remainders in compact extensions, Soviet Math. Dokl. 6 (1965), 136-140.

6. K. Menger, Über umfassendste n-dimensionale Mengen, Proc. Ned. Akad. Wetenschap. 29 (1926), 1125-1128.

7. J. Nagata, On the countable sum of zero-dimensional metric spaces, Fund. Math. 48 (1960), 1-14.

8. - Modern dimension theory, Wiley, New York, 1965.

9. E. G. Sklyarenko, On dimensional properties of infinite-dimensional spaces, Amer. Math. Soc. Translations (2) 21 (1962), 35-50.

UNIVERSITY OF KANSAS 